\title{
Promoting appropriate medical discharges over the weekend - is 7-day working a reality?
}

\author{
Authors: Alex Fulton, Sam Heward, Sally Briggs, Jennifer Beynon and Jayne Little*
}

\section{Introduction}

Timely discharge of patients is important for patient safety and satisfaction as well as patient flow and health economics. Improving hospital discharges and promotion of a 7-day service are key priorities of NHS England. Discharging medical patients from hospital over the weekend can be challenging due to both intrinsic and extrinsic issues. We aimed to explore these issues, identify key areas for improvement and increase the number or patients appropriately discharged over the weekend at a major acute teaching hospital in Manchester.

\section{Materials and methods}

Routinely collected data was explored to calculate the following information relating to discharges of medical patients over a 12 month period from 1 September 2017 to 31 August 2018 (from any ward other than the acute medical unit (AMU)):

$>$ number of discharges by day

> number of weekend discharges per week (defined as 17.00 on Friday to 9.00 on Monday)

> proportion of weekly discharges occurring over the weekend by month.

The same information was calculated on a 'pilot' ward (endocrine and general medicine).

A process mapping session including all relevant stakeholders on the pilot ward (therapist, ward clerk, ward manager, junior doctor and consultant, pharmacist and manager) allowed identification of key areas for improvement.

Criteria-led discharge forms (CLD) and weekend handover pro formas were introduced as part of PDSA cycles while collecting the following measures:

> number of discharges per weekend (primary outcome measure)

> number of Fridays when weekend discharges are considered and number of CLD forms and handover pro formas completed (process measures)

$>$ junior doctor satisfaction (balancing measure).

\section{Results and discussion}

There were 5,461 medical discharges over 12 months, 557 (10.6\%) of which occurred over the weekend. The greatest proportion of

Authors: Manchester University NHS Foundation Trust ${ }^{*} \mathrm{RCP}$ chief registrar

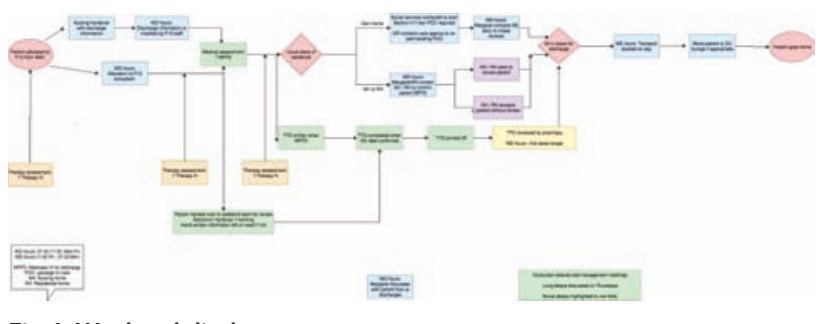

Fig 1. Weekend discharges

discharges occurred on a Wednesday, Thursday and Friday (1,036 (19.0\%), 1,043 (19.0\%) and 1,046 (19.2\%) respectively). There did not appear to be any seasonal variation or other patterns in the number or proportion of patient discharged at the weekend (with the exception of a rise in proportion of weekend discharges in relation to the Christmas period). These findings were also reflected in the 'pilot' ward. Process mapping identified the following key areas for improvement (see Fig 1).

1. Clear identification of patients suitable for weekend discharge.

2. Communication of this information to the weekend team.

3. Empowerment of the weekend team to discharge.

4. Appropriate allocation of patients to the ward (those without complex care needs).

The development of a paper handover pro forma including a checklist of the following tackled items 1 and 2 above.

$>$ Completion of TTO (to take out).

$>$ Outstanding investigations.

> Weekend requirements (nurse-led discharge / medical review / therapy assessment).

CLD forms aimed to address item 3 by empowering nursing and junior medical staff to discharge patients appropriately.

There has not yet been a significant change in the number of patients discharged over the weekend and few suitable patients have been identified for CLD despite team engagement. We aim to address 'key area' 4 above as part of the next PDSA cycle.

\section{Conclusion}

Despite team engagement we have found it challenging to increase the number of medical patients discharged over the weekend. This may be due to the significant numbers of patients having complex social and care needs and requires further exploration. 